\title{
Atypical Presentation of Malignant Melanoma in a 16 Months Old Child
}

\author{
Liv Schoellhammer ${ }^{1, *}$, Torben Stamm Mikkelsen ${ }^{2}$, Henrik Schmidt ${ }^{3}$, Lene Birk-Soerensen ${ }^{1}$ \\ ${ }^{1}$ Department of Plastic surgery, Aalborg University Hospital, 9000 Aalborg Denmark \\ ${ }^{2}$ Department of Pediatrics, Herning Hospital, 7400 Herning, Denmark \\ ${ }^{3}$ Department of Oncology, Aarhus University Hospital, 8200 Aarhus N, Denmark \\ *Corresponding author: liv.schoellhammer@gmail.com
}

Received January 21, 2015; Revised February 03, 2015; Accepted February 09, 2015

\begin{abstract}
Malignant melanoma in childhood and adolescence is rare but incidences have been seen to rise. Prognostic factors, risk factors and prognosis have similarities to melanoma in adulthood but paediatric melanoma presents a diagnostic challenge as it often lacks the classical features of adult pigmented malignant melanoma and histologically is difficult to diagnose. This case report describes a clinically atypical presentation of melanoma on the thigh of a 16 month old boy. Initially seen at five months old by a dermatologist were a punch biopsy of the element was made with histological diagnosis; unusual congenital nevus. The element hereafter evolved and at the time of diagnosis measured $7.5 \mathrm{~mm}$ in Breslow thickness. Sentinel node biopsy and full body scans were made with no sign of metastasis. Like other cases reported, the melanoma did not exhibit the classical characteristics of the ABCD criteria which may have played a part in delaying diagnosis. Studies suggest that delayed diagnosis in children is not uncommon and younger patients present with more advanced disease at the time of diagnosis. To facilitate earlier recognition of melanoma in children additions to the conventional ABCD criteria have been suggested. This report illustrates that malignant melanoma should always be taken into consideration when presented with lesions of the skin and that a high level of clinical awareness and suspicion is required in order to hasten diagnosis and maybe improve prognosis in cutaneous malignant melanoma in children and adolescents.
\end{abstract}

Keywords: melanoma, nevus, pigmented, child, dermatology, adolescent

Cite This Article: Liv Schoellhammer, Torben Stamm Mikkelsen, Henrik Schmidt, and Lene Birk-Soerensen, "Atypical Presentation of Malignant Melanoma in a 16 Months Old Child.” Journal Name, vol. 3, no. 1 (2015): 79-81. doi: 10.12691/ajmcr-3-3-7.

\section{Introduction}

Malignant melanoma in childhood and adolescence is rare. Melanoma represents 1-3\% of cancers diagnosed in patients younger than 20 years and only $0.9 \%$ in children under the age of 15 [1,2]. In a study from 2002, only 1.3\% of new melanoma cases diagnosed in USA from 1973 to 1996 were younger than 20 years [3]. Though rare, the incidence of childhood melanoma has been rising 2-2.9\% per year in USA from 1973-2001 and has been seen to double in Sweden from 1973-1992 [4,5,6]. Reported incidences in Australia and in the United States, are up to 30 and 14 per million respectively in adolescents. In Sweden the incidence increased to 5 per million in 1992, though in the following years decreasing to 3.6 per million in 2002 [2,6,7].

Looking at gender distribution, there is a female predominance in melanoma patients with girls comprising $60 \%$ in the age group 10 to 19 . Only in the youngest children (1 to 4 years) males predominate and the overall female/male ratio is $1.6[8,9,11]$. The most common primary sites of cutaneous melanoma in children are the trunk and lower extremities, except for congenital and infantile melanoma which most often occurs in the head and neck area $[3,4]$.

Histologically pediatric melanomas, like melanoma in adulthood, can the classified into subtypes. The most common subtypes described in literature are superficial spreading and nodular melanoma. Also acral lentiginous, lentigo maligna, desmoplastic and neuroptropic melanomas in addition to small cell melanoma, melanoma with Spitzoid features, malignant blue naevus and animal type melanoma has been described but are rare. Lentigo maligna melanoma and desmoplastic melanoma being exceedingly rare as they arise from cronic sun exposure and generally only presents in childhood in patients with xeroderma pigmentosum [7]. Lesions diagnosed as a melanoma but unclassifiable subtypes also represent a large part of melanomas in childhood. In a study from 2009 unclassified and Spizoid melanomas comprised 47\% of the melanomas in patients younger than 10 years, followed to severely atypical ambiguous lesions (32\%) [10].

The most significant prognostic factors for pediatric melanoma, like in adulthood, are: melanoma thickness, ulceration and stage at diagnosis (lymph node involvement and distant metastasis) [1,9]. Studies have shown a 5-year survival according to disease stage of 
$100 \%$ for in situ disease, 93-96\% for localized disease, 68$77 \%$ for regional metastatic disease and $11-57 \%$ for distant metastatic disease [1]. A recent study by Averbook et al. found that children aged $\leq 10$ years with invasive melanoma present with more advanced disease at time of diagnosis but also demonstrate a trend towards increased survival compared with older pediatric cohorts [9].

Risk factors for childhood melanoma are similar to those in adulthood, and include sun sensitivity characterized by light skin, light or red hair, heavy facial freckling, inability to tan and a tendency to sunburn. Also sun exposure, multiple melanocytic nevi, dysplastic nevus syndrome and a family history of melanoma are risk factors. Furthermore in pediatric patients, xeroderma pigmentosum, giant congenital melanocytic nevus and immunosuppression are associated with an increased risk of malignant melanoma [1,5,7]. Risk factors specific for melanoma of infancy and early childhood have not been identified.

Pediatric melanoma, especially in prepubertal children, presents a diagnostic challenge. Malignant melanoma in children often lacks the classical features of adult pigmented malignant melanoma and histologically it is difficult to diagnose, particularly in children within the first year of life $[7,11]$.

In this report we present a case of thick cutaneous melanoma in a 16 month old Scandinavian boy. We look in to risk factors, differential diagnosis and prognostic factors in childhood melanoma. We also discuss a possible delay in diagnosis and suggested additions to the conventional ABCD criteria designed to facilitate earlier recognition of melanoma in children.

\section{Case}

A 16 month old boy was referred to the emergency room by a general practitioner on the suspicion of an abscess on the thigh. Within the first months after birth the patient had developed a small element proximal on his right thigh. At five months he had been examined by a dermatologist. The clinical impression was cutaneous mastocytoma, hamartoma or nevus. A punch biopsy was made and the histological diagnosis was unusual congenital nevus with focal sclerosis and spindle cell features. Over the next 12 months the element grew in size and thickness.

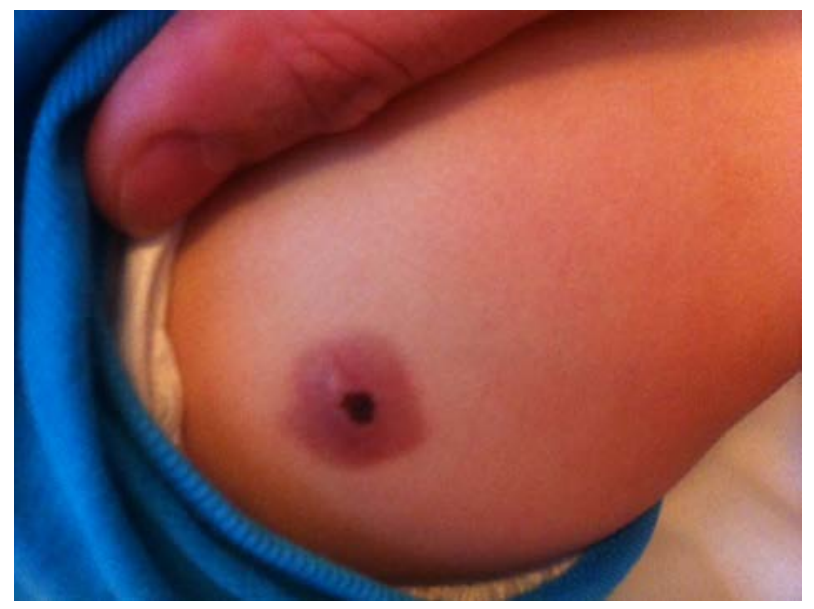

Figure 1. Clinical presentation of malignant melanoma on the right thigh
When examined at the emergency room the patient presented with an element measuring 17x19 mm. The element was ulcerated centrally with telangiectasias and a bluish color in the circumference (Figure 1). The lesion was firm but not tender.

The boy was previously healthy and had had a normal birth and early childhood development. No family history of malignant melanoma was present. The patient had fair skin and blue eyes and he had not been exposed much to sunlight, neither had he experienced any sunburns. On examination of the skin only a few nevi were found and none were found to be suspicious.

The element was primarily excised at a $5 \mathrm{~mm}$ distance. Histological examination was made at three different institutions of pathology in Denmark and in the United States. In categorizing the tumor stains were performed and found the cells to be positive for MLA, negative for CD20 and CD3 with an almost diffuse reaction for HMB45. Proliferations fraction was about 5\% estimated from a Ki67-staining. Also a NeoSITE 5 probe (6p25, 9p21/CEN9, 8q24 and 11q13) fluorescence in situ hybridization (FISH) test and SNP-microarray genetic analysis were performed. The FISH test exhibited signal within normal ranges but does not preclude a diagnosis of melanoma as it can be seen in $10-20 \%$ of conventional melanomas and this percentage may even be higher in unusual variants of melanoma. The pathologists agreed on the diagnosis: Severely atypical melanocytic tumor best considered as childhood melanoma, subtype unclassified, level 5, Breslow thickness $7.5 \mathrm{~mm}$. The surface was ulcerated and there was seen up to 7 mitosis per $\mathrm{mm}^{2}$.

Re-excision was made in a $15 \mathrm{~mm}$ margin from the primary scar and a sentinel node biopsy procedure was performed. Two lymph nodes were removed from the right groin, none of them showing signs of metastasis. Due to activity under the abdominal fascia when performing the lymph scintigraphy, a computed tomography scan of the thorax and a magnetic Resonance Imaging scan of the abdomen were made, both without signs of metastases. The patient was referred to the department of pediatric oncology for further follow-up. The follow-up program consists of observation with clinical examination every 3-6 months for 5 years.

\section{Discussion}

Compared with melanoma in adults, studies suggest that delayed diagnosis in children is not uncommon. Furthermore, younger patients present with more advanced disease at the time of diagnosis [2,9,10]. A contributing factor to this may be that melanoma in this age group often do not present with the conventional signs as seen in adults. In a cohort study from USA from 19842009 with 70 patients under the age of 20, up to $60 \%$ of the patients under 10 years, the melanoma did not exhibit the classical characteristics of the ABCD criteria and 77\% were amelanotic. Though clinical presentation varied greatly lesional evolution, especially increased size, was nearly universal [10].

The most common clinical differential diagnosis for melanoma in children includes pyogenic granulomas, warts and benign nevi including Spitz nevi $[7,10]$. Also hemangiomas are not uncommon in infants and these 
benign vascular tumors often grow within the first year of life and can develop into bleeding ulcers. Histological examination also presents a challenge as childhood melanoma microscopically resembles Spitz nevi, dysplastic nevi, traumatized nevi, halo nevi and some congenital melanocytic nevi [1].

To facilitate earlier recognition of melanoma in children additions to the conventional ABCD criteria have been suggested to include: amelanotic, bleeding, color uniformity, de novo lesion, any diameter and evolution, $[10,12]$.

In the presented case the lesion did not show the clinical signs of malignancy given by the ABCD criteria excluding diameter. It did, however, present several of the suggested additional criteria: amelanotic, bleeding and evolution.

This report illustrates that malignant melanoma should always be taken into consideration when presented with lesions of the skin. In this case the patient had no risk factors, was of an age where melanoma is very unlikely and lived in a country with limited sun exposure. This exemplifies that a high level of clinical awareness and suspicion is required in order to hasten diagnosis and maybe improve prognosis in cutaneous malignant melanoma in children and adolescents

\section{Funding}

None.

\section{Statement of Competing Interests}

The authors have no competing interests.

\section{Reference List}

[1] Jen M, Murphy M, Grant-Kels JM. Childhood melanoma. Clin Dermatol 2009 November; 27 (6): 529-36.

[2] Pappo AS. Melanoma in children and adolescents. Eur J Cancer 2003 December; 39 (18): 2651-61.

[3] Hamre MR, Chuba P, Bakhshi S, Thomas R, Severson RK. Cutaneous melanoma in childhood and adolescence. Pediatr Hematol Oncol 2002 July; 19 (5): 309-17.

[4] Strouse JJ, Fears TR, Tucker MA, Wayne AS. Pediatric melanoma: risk factor and survival analysis of the surveillance, epidemiology and end results database. J Clin Oncol 2005 July 20; 23 (21): 4735-41.

[5] Wong JR, Harris JK, Rodriguez-Galindo C, Johnson KJ. Incidence of childhood and adolescent melanoma in the United States: 19732009. Pediatrics 2013 May; 131 (5): 846-54.

[6] Karlsson PM, Fredrikson M. Cutaneous malignant melanoma in children and adolescents in Sweden, 1993-2002: the increasing trend is broken. Int J Cancer 2007 July 15; 121 (2): 323-8.

[7] Brenn T, McKee PH. Melanoma in children and adolescents. Diagnostic Histopathology 2008 January 21; 14 (1).

[8] Han D, Zager JS, Han G, Marzban SS, Puleo CA, Sarnaik AA, Reed D, Messina JL, Sondak VK. The unique clinical characteristics of melanoma diagnosed in children. Ann Surg Oncol 2012 November; 19 (12): 3888-95.

[9] Averbook BJ, Lee SJ, Delman KA, Gow KW, Zager JS, Sondak VK, Messina JL, Sabel MS, Pittelkow MR, Ecker PM, Markovic SN, Swetter SM, Leachman SA, Testori A, Curiel-Lewandrowski C, Go RS, Jukic DM, Kirkwood JM. Pediatric melanoma: analysis of an international registry. Cancer 2013 November 15; 119 (22): 4012-9.

[10] Cordoro KM, Gupta D, Frieden IJ, McCalmont T, Kashani-Sabet M. Pediatric melanoma: results of a large cohort study and proposal for modified ABCD detection criteria for children. J Am Acad Dermatol 2013 June; 68 (6): 913-25.

[11] Moscarella E, Piccolo V, Argenziano G, Lallas A, Longo C, Castagnetti F, Pizzigoni S, Zalaudek I. Problematic lesions in children. Dermatol Clin 2013 October; 31 (4): 535-47, vii.

[12] Abbasi NR, Shaw HM, Rigel DS, Friedman RJ, McCarthy WH, Osman I, Kopf AW, Polsky D. Early diagnosis of cutaneous melanoma: revisiting the ABCD criteria. JAMA 2004 December 8; 292 (22): 2771-6. 